J. Korean Math. Soc. 51 (2014), No. 5, pp. 1075-1088

http://dx.doi.org/10.4134/JKMS.2014.51.5.1075

\title{
PACKING DIMENSIONS OF GENERALIZED RANDOM MORAN SETS
}

\author{
Xin Tong, Yue-li Yu, and XiaO-Jun ZhaO
}

\begin{abstract}
We consider random fractal sets with random recursive constructions in which the contracting vectors have different distributions at different stages. We prove that the random fractal associated with such construction has a constant packing dimension almost surely and give an explicit formula to determine it.
\end{abstract}

\section{Introduction}

There has been considerable interest in fractals, both in their occurrence in the sciences, and in their mathematical theory. A wide class of fractal sets are generated by recursive constructions or iterated function systems (IFS). The recursive construction was first introduced by Moran in [17] and systematically studied by Hutchinson [11].

In the 1980s, Falconer [3], Graf [6], Mauldin and Williams [16] investigated random fractal sets by randomizing each step in Moran's deterministic constructions. They studied the geometric properties of such random constructions and obtained the Hausdorff dimensions of such sets. Later Graf, Mauldin and Williams [16], Arbeiter and Patzschke [2], Olsen [18] considered the random measures associated with the random recursive constructions. Similar random recursive constructions and random sets were studied by Pesin and Weiss [22], Kifer $[13,14]$ employing the thermodynamic formalism for random subshifts of finite type. All the papers mentioned above work in the settings when the probability distributions of contracting vectors at each stage of the construction are independent and identically distributed. Liu, Wen and $\mathrm{Wu}[15]$ generalized the works of Falconer [3], Graf [6], Mauldin and Williams [16] to the case that at each stage, the contracting vectors were not identically distributed. They proved that the random fractal associated with such construction has a constant Hausdorff dimension almost surely and gave an explicit formula to determine it. For more results, see $[1,5,7,8,9,10,12,19,20,21]$.

Received February 27, 2014.

2010 Mathematics Subject Classification. Primary 60D05; Secondary 28A80, 28 A78.

Key words and phrases. generalized random Moran construction, packing dimension, negative moment. 
The main aim of this paper is to determine the packing dimensions of the generalized random Moran sets in [15].

Before introducing the generalized random construction, we give some notation.

Let $\left\{n_{k}, k \geq 1\right\}$ be a sequence of positive integers with $n_{k} \geq 2$ for any $k \geq 1$. For any $k \geq 1$, let

$$
\begin{aligned}
I_{k} & =\left\{\sigma=\left(\sigma_{1}, \sigma_{2}, \ldots, \sigma_{k}\right): 1 \leq \sigma_{j} \leq n_{j}, 1 \leq j \leq k\right\}, \\
I_{\infty} & =\left\{\sigma=\left(\sigma_{1}, \sigma_{2}, \ldots, \sigma_{k}, \ldots\right): 1 \leq \sigma_{j} \leq n_{j}, 1 \leq j<\infty\right\}, \\
I_{*} & =\bigcup_{k=1}^{\infty} I_{k} .
\end{aligned}
$$

Besides, we give other notation:

(1) $\sigma \mid k=\left(\sigma_{1}, \sigma_{2}, \ldots, \sigma_{k}\right)$ where $\sigma \in I_{\infty}$ or $\sigma \in I_{l}$ for $l \geq k$,

(2) $\sigma * \tau=\left(\sigma_{1}, \sigma_{2}, \ldots, \sigma_{k}, \tau_{1}, \tau_{2}, \ldots, \tau_{l}\right)$ for $\sigma=\left(\sigma_{1}, \sigma_{2}, \ldots, \sigma_{k}\right) \in I_{k}$ and $\tau=\left(\tau_{1}, \tau_{2}, \ldots, \tau_{l}\right) \in I_{l}$

(3) $|\sigma|$ denotes the length of $\sigma \in I_{*}$. That is $|\sigma|=k$ if $\sigma \in I_{k}$. Let $I_{0}$ denote the empty set $\emptyset$.

Now we consider the following generalized random construction.

Let $J$ be a nonempty compact subset of $\mathbb{R}^{d}$ such that $\overline{\operatorname{int}(J)}=J$, where $\operatorname{int}(J)$ and $\bar{J}$ denote the interior and the closure of $J$ respectively. Let $\left\{n_{k}\right\}_{k \geq 1}$ be a sequence of positive integers satisfying $n_{k} \geq 2$ for any $k \geq 1$.

Let $(\Omega, \mathcal{F}, P)$ be a complete probability space and $\mathcal{J}=\left\{J_{\sigma}: \sigma \in I_{*}\right\}$ be a family of random compact subsets of $\mathbb{R}^{d}$ satisfying:

(1) $J_{\emptyset}(\omega)=J$ for almost all $\omega \in \Omega$; for any $\sigma \in I_{*}$ and almost all $\omega \in \Omega$, $J_{\sigma}(\omega)$ is geometrically similar to $J$.

(2) For almost all $\omega \in \Omega$ and for every $k \geq 0$, if $\sigma \in I_{k}$, then $J_{\sigma * 1}(\omega)$, $J_{\sigma * 2}(\omega), \ldots, J_{\sigma * n_{k+1}}(\omega)$ is a sequence of non-overlapping subsets of $J_{\sigma}(\omega)$. ( $E$ and $F$ non-overlapping means $\operatorname{int}(E) \cap \operatorname{int}(F)=\emptyset$.)

(3) Random vectors $\left\{\left(T_{\sigma * 1}, \ldots, T_{\sigma * n_{|\sigma|+1}}\right): \sigma \in I_{*}\right\}$ are independent. For every $k \geq 0$, the random vectors $\left\{\left(T_{\sigma * 1}, \ldots, T_{\sigma * n_{k+1}}\right): \sigma \in I_{k}\right\}$ have the same distribution, where $T_{\sigma * j}(\omega)$ is the ratio of the diameter of $J_{\sigma * j}(\omega)$ to the diameter of $J_{\sigma}(\omega), 1 \leq j \leq n_{|\sigma|+1}$.

(4) There exists $\delta_{1}>0$ such that for almost all $\omega \in \Omega$, for every $k \geq 0$ and $\sigma \in I_{k}, T_{\sigma * j}(\omega) \geq \delta_{1}$ for any $1 \leq j \leq n_{k+1}$.

The family $\mathcal{J}$ is called a generalized random Moran construction. Let

$$
K(\omega)=\bigcap_{k=0}^{\infty} \bigcup_{\sigma \in I_{k}} J_{\sigma}(\omega),
$$

and the set $K(\omega)$ is called the generalized random Moran set associated with the family $\mathcal{J}$. 
For any $k \geq 1$, let $s_{k}$ satisfy the equation

$$
\mathbb{E} \sum_{\sigma \in I_{k}} \prod_{i=1}^{k} T_{\sigma \mid i}^{s_{k}}=1
$$

then $s_{k}$ is called the $k$-th predimension of the random set $K(\omega)$.

Let

$$
s_{*}=\liminf _{k \rightarrow \infty} s_{k}, \quad s^{*}=\limsup _{k \rightarrow \infty} s_{k}
$$

Liu, Wen and $\mathrm{Wu}[15]$ showed that for almost all $\omega \in \Omega$,

$$
\operatorname{dim}_{H} K(\omega)=s_{*} .
$$

In this paper, we obtain the following result:

Theorem 1.1. For almost all $\omega \in \Omega$,

$$
\operatorname{dim}_{P} K(\omega)=s^{*} \text {. }
$$

In [15], the dimensional result was obtained by defining a martingale associated with the geometric construction and choosing a suitable parameter. The proof of the results in [15] is based on the existence of the moments of all orders and a common bound of these moments. In this paper, in order to get the packing dimensions of the generalized random Moran sets, we shall use the negative moment instead, which is considerably different from [15].

The paper is organized as follows. We give some notation and preliminaries in Section 2. In Section 3, we first define the so-called $J_{r}$-packing and $J$-packing dimensions for the generalized Moran sets. And we prove that the upper $J$ packing dimensions are equivalent to upper box dimensions. We prove the main theorem in the last section.

\section{Preliminaries}

First, we recall the definitions of packing measure and packing dimension.

Let $E$ be a non-empty bounded subset of $\mathbb{R}^{d}$. For $\delta>0$, a $\delta$-packing of $E$ is defined as a finite or countable collection of disjoint balls $\left\{B_{i}\right\}$ of radii at most $\delta$ with centers in $E$. For any $s>0$, we first define $\mathcal{P}_{0, \delta}^{s}$ by

$$
\mathcal{P}_{0, \delta}^{s}(E)=\sup \left\{\sum_{i=1}^{\infty}\left|B_{i}\right|^{s}:\left\{B_{i}\right\} \text { is a } \delta \text {-packing of } \mathrm{E}\right\},
$$

where $\left|B_{i}\right|$ denotes the diameter of $B_{i}$. Then $\mathcal{P}_{0, \delta}^{s}(E)$ is non-increasing as $\delta$ decreases by the definition of $\mathcal{P}_{0, \delta}^{s}(E)$. We take the limit

$$
\mathcal{P}_{0}^{s}(E)=\lim _{\delta \rightarrow 0} \mathcal{P}_{0, \delta}^{s}(E) \text {. }
$$

The $s$-dimensional packing measure of $E$ is defined by

$$
\mathcal{P}^{s}(E)=\inf \left\{\sum_{i=1}^{\infty} \mathcal{P}_{0}^{s}\left(E_{i}\right): E \subset \bigcup_{i=1}^{\infty} E_{i}\right\} .
$$


The packing dimension of $E$ is defined by

$$
\operatorname{dim}_{P}(E)=\inf \left\{s: \mathcal{P}^{s}(E)=0\right\}=\sup \left\{s: \mathcal{P}^{s}(E)=\infty\right\} .
$$

Next, we recall the definition of upper Minkowski(or box) dimension.

Let $N_{r}(E)$ be the smallest number of closed balls of radii $r$ that can cover $E$. The upper box dimensions of $E$ are defined by

$$
\overline{\operatorname{dim}}_{B}(E)=\limsup _{r \rightarrow 0} \frac{\log N_{r}(E)}{-\log r} .
$$

An equivalent definition of upper box dimension of a rather different nature involves the $d$-dimensional volume of the $r$-neighbourhood or $r$-parallel body $E_{r}$ of $E$, given by

$$
E_{r}=\left\{x \in R^{d}:|x-y| \leq r \text { for some } y \text { in } E\right\}
$$

Then for $E \subset \mathbb{R}^{d}$,

$$
\overline{\operatorname{dim}}_{B}(E)=d-\liminf _{r \rightarrow 0} \frac{\log \mathscr{L}^{d}\left(E_{r}\right)}{\log r},
$$

where $\mathscr{L}^{d}$ is the $d$-dimensional volume or $d$-dimensional Lebesgue measure.

By Corollary 3.9 in [4], the packing dimension of a set is equal to the upper boxing dimension of it in the following situation.

Lemma 2.1. Let $E$ be a non-empty compact subset of $\mathbb{R}^{d}$. If for an arbitrary open set $V$ we have $\overline{\operatorname{dim}}_{B}(E)=\overline{\operatorname{dim}}_{B}(E \cap V)$, then $\operatorname{dim}_{P}(E)=\overline{\operatorname{dim}}_{B}(E)$

\section{Equivalent definition}

In this section, we shall give an equivalent definition of the upper box dimension for the generalized random Moran sets. We first consider the deterministic Moran sets with the same constructions. Now let us recall the definition of Moran sets with deterministic constructions. Let $J$ be a nonempty compact subset of $\mathbb{R}^{d}$ such that $\overline{\operatorname{int}(J)}=J$. And let $\left\{n_{k}\right\}_{k \geq 1}$ be a sequence of positive integers satisfying $n_{k} \geq 2$ for any $k \geq 1$. We call the subcollection $\mathcal{J}=\left\{J_{\sigma}: \sigma \in I_{*}\right\}$ of $\mathbb{R}^{d}$ a Moran construction, if

(1) $J_{\emptyset}=J$ and $J_{\sigma}$ is geometrically similar to $J$.

(2) For every $k \geq 0$, if $\sigma \in I_{k}$, then $J_{\sigma * 1}, J_{\sigma * 2}, \ldots, J_{\sigma * n_{k+1}}$ is a sequence of non-overlapping subsets of $J_{\sigma}$. ( $E$ and $F$ non-overlapping means $\operatorname{int}(E) \cap \operatorname{int}(F)=\emptyset$.)

(3) For every $k \geq 0, \sigma \in I_{k}$ and $1 \leq j \leq n_{k+1}, T_{\sigma * j}$ is the ratio of the diameter of $\bar{J}_{\sigma * j}$ to the diameter of $J_{\sigma}$.

Let

$$
K=\bigcap_{k=0}^{\infty} \bigcup_{\sigma \in I_{k}} J_{\sigma}
$$


Then the nonempty compact set $K$ is called the generalized Moran set associated with $\mathcal{J}$.

In our cases, we assume there exist $0<\delta_{1}<\delta_{2}<1$ such that $\delta_{1} \leq T_{\sigma * j} \leq \delta_{2}$ for any $\sigma \in I_{*}$ and any $1 \leq j \leq n_{|\sigma|+1}$.

Definition 3.1. For any $r>0$, let

$$
\widetilde{\mathcal{J}}_{r}=\left\{J_{\sigma}:\left|J_{\sigma}\right| \leq r<\left|J_{\sigma \mid(|\sigma|-1)}\right|, \sigma \in I_{*}\right\},
$$

and call the family $\widetilde{\mathcal{J}}_{r}$ a $J_{r}$-packing of $K$.

It is clear that $\widetilde{\mathcal{J}}_{r}$ is also a finite nonoverlapping $r$-covering of $K$.

For $s \geq 0$, we define

$$
\mathcal{P}_{\mathcal{J}}^{s}(K)=\limsup _{r \rightarrow 0} \sum_{\sigma: J_{\sigma} \in \widetilde{\mathcal{J}}_{r}}\left|J_{\sigma}\right|^{s},
$$

and define the upper $J$-packing dimension

$$
\overline{\operatorname{dim}}_{\mathcal{J}}(K)=\inf \left\{s: \mathcal{P}_{\mathcal{J}}^{s}(K)=0\right\}=\sup \left\{s: \mathcal{P}_{\mathcal{J}}^{s}(K)=\infty\right\}
$$

The following propositions will show the equivalence of the upper $J$-packing dimension and the upper box dimension.

Proposition 3.2. Let $M_{r}(K)$ be the number of sets $J_{\sigma}$ in $\widetilde{\mathcal{J}}_{r}$. Then

$$
\overline{\operatorname{dim}}_{\mathcal{J}}(K)=\limsup _{r \rightarrow 0} \frac{\log M_{r}(K)}{-\log r} .
$$

Proof. For any $\alpha>\overline{\operatorname{dim}}_{\mathcal{J}}(K)$, by the definition of the upper $J$-packing dimension, we have

$$
\mathcal{P}_{\mathcal{J}}^{\alpha}(K)=\limsup _{r \rightarrow 0} \sum_{\sigma: J_{\sigma} \in \widetilde{\mathcal{J}}_{r}}\left|J_{\sigma}\right|^{\alpha}=0 .
$$

Since $T_{\sigma * j} \geq \delta_{1}$ for all $\sigma \in I_{*}$ and all $1 \leq j \leq n_{|\sigma|+1}$, we have $\left|J_{\sigma}\right| \geq \delta_{1} r$ for any $J_{\sigma} \in \widetilde{\mathcal{J}}_{r}$. Thus

$$
\sum_{J_{\sigma} \in \widetilde{\mathcal{J}}_{r}}\left|J_{\sigma}\right|^{\alpha} \geq M_{r}(K)\left(\delta_{1} r\right)^{\alpha}
$$

which implies

$$
\limsup _{r \rightarrow 0} \frac{\log M_{r}(K)}{-\log r} \leq \alpha .
$$

Since $\alpha>\overline{\operatorname{dim}}_{\mathcal{J}}(K)$ is arbitrary, it follows that

$$
\overline{\operatorname{dim}}_{\mathcal{J}}(K) \geq \limsup _{r \rightarrow 0} \frac{\log M_{r}(K)}{-\log r} .
$$

On the other hand, for any $0 \leq t<\beta<\overline{\operatorname{dim}}_{\mathcal{J}}(K)$, we have

$$
\mathcal{P}_{\mathcal{J}}^{\beta}(K)=\limsup _{r \rightarrow 0} \sum_{\sigma: J_{\sigma} \in \widetilde{\mathcal{J}}_{r}}\left|J_{\sigma}\right|^{\beta}=\infty
$$


by the definition of $\overline{\operatorname{dim}}_{\mathcal{J}}(K)$. Therefore the inequality

$$
\sum_{\sigma: J_{\sigma} \in \widetilde{\mathcal{J}}_{r}}\left|J_{\sigma}\right|^{\beta}>1
$$

holds for a sequence of positive real numbers $r \rightarrow 0$. For any positive $r$ satisfying (5), let $n_{k}$ be the number of sets $J_{\sigma} \in \widetilde{\mathcal{J}}_{r}$ with $\delta_{2}^{k+1}<\left|J_{\sigma}\right| \leq \delta_{2}^{k}$. Then by $(5)$,

$$
\sum_{k \geq 0} n_{k} \delta_{2}^{k \beta} \geq \sum_{\sigma: J_{\sigma} \in \widetilde{\mathcal{J}}_{r}}\left|J_{\sigma}\right|^{\beta}>1
$$

We claim that there is a positive integer $k_{r}$ satisfying

$$
n_{k_{r}} \geq \delta_{2}^{-k_{r} t}\left(1-\delta_{2}^{\beta-t}\right)
$$

Otherwise $n_{k} \delta_{2}^{k t}<1-\delta_{2}^{\beta-t}$ for all $k \geq 0$, then

$$
1<\sum_{k \geq 0} n_{k} \delta_{2}^{k \beta}=\sum_{k \geq 0} n_{k} \delta_{2}^{k t} \delta_{2}^{k(\beta-t)} \leq \sum_{k \geq 0}\left(1-\delta_{2}^{\beta-t}\right) \delta_{2}^{k(\beta-t)}=1,
$$

which is a contradiction. For any $J_{\sigma} \in \widetilde{\mathcal{J}}_{r}$ with $\delta_{2}^{k+1}<\left|J_{\sigma}\right| \leq \delta_{2}^{k}$, we have $J_{\sigma} \in \widetilde{\mathcal{J}}_{\delta_{2}^{k}}$ since $T_{\sigma} \leq \delta_{2}$. So

$$
M_{\delta_{2}^{k_{r}}}(K) \geq n_{k_{r}} \geq \delta_{2}^{-k_{r} t}\left(1-\delta_{2}^{\beta-t}\right) .
$$

Since $n_{k_{r}} \geq 1$, there exists at least one set $J_{\sigma} \in \widetilde{\mathcal{J}}_{r}$ with $\delta_{2}^{k_{r}+1}<\left|J_{\sigma}\right| \leq \delta_{2}^{k_{r}}$. So $\delta_{2}^{k_{r}+1}<\left|J_{\sigma}\right| \leq r$, which implies $k_{r} \rightarrow \infty$ as $r \rightarrow 0$. Thus

$$
\limsup _{r \rightarrow 0} \frac{\log M_{r}(K)}{-\log r} \geq \limsup _{k_{r} \rightarrow \infty} \frac{\log M_{\delta_{2}^{k_{r}}}(K)}{-\log \delta_{2}^{k_{r}}} \geq \limsup _{k_{r} \rightarrow \infty} \frac{\log \delta_{2}^{-k_{r} t}\left(1-\delta_{2}^{\beta-t}\right)}{-\log \delta_{2}^{k_{r}}}=t .
$$

Since $t<\overline{\operatorname{dim}}_{\mathcal{J}}(K)$ is arbitrary, it follows that

$$
\overline{\operatorname{dim}}_{\mathcal{J}}(K) \leq \limsup _{r \rightarrow 0} \frac{\log M_{r}(K)}{-\log r} .
$$

Proposition 3.3. Let $M_{r}(K)$ be the number of sets $J_{\sigma}$ in $\widetilde{\mathcal{J}}_{r}$. Then

$$
\limsup _{r \rightarrow 0} \frac{\log M_{r}(K)}{-\log r}=\overline{\operatorname{dim}}_{B}(K) .
$$

Proof. By the definition of upper box dimension, it is sufficient to show that

$$
\limsup _{r \rightarrow 0} \frac{\log M_{r}(K)}{-\log r}=d-\liminf _{r \rightarrow 0} \frac{\log \mathscr{L}^{d}\left(K_{r}\right)}{\log r},
$$

where $K_{r}$ is the $r$-parallel body of $K$ and $\mathscr{L}^{d}$ denote the Lebesgue measure. It is clear that $\bigcup_{J_{\sigma} \in \widetilde{\mathcal{J}}_{r}} J_{\sigma} \subset K_{r}$. Note that $J_{\sigma}$ is geometrically similar to $J$. We 
obtain $\mathscr{L}^{d}\left(J_{\sigma}\right) \geq \frac{\left(\delta_{1} r\right)^{d} \mathscr{L}^{d}(J)}{|J|^{d}}$ for any $J_{\sigma} \in \widetilde{\mathcal{J}}_{r}$, since $\left|J_{\sigma}\right| \geq \delta_{1} r$ for any $J_{\sigma} \in \widetilde{J}_{r}$. Write $C_{d}=\frac{\delta_{1}^{d} \mathscr{L}^{d}(J)}{|J|^{d}}$. Hence

$$
\mathscr{L}^{d}\left(K_{r}\right) \geq \mathscr{L}^{d}\left(\bigcup_{J_{\sigma} \in \widetilde{\mathcal{J}}_{r}} J_{\sigma}\right) \geq M_{r}(K) r^{d} C_{d} .
$$

Thus

$$
\limsup _{r \rightarrow 0} \frac{\log M_{r}(K)}{-\log r} \leq d-\liminf _{r \rightarrow 0} \frac{\log \mathscr{L}^{d}\left(K_{r}\right)}{\log r} .
$$

On the other hand, for any $J_{\sigma} \in \widetilde{\mathcal{J}}_{r}$, let

$$
G_{\sigma}=\left\{x: d\left(x, J_{\sigma}\right) \leq r\right\}
$$

Since

$$
\bigcup_{\sigma: J_{\sigma} \in \widetilde{\mathcal{J}}_{r}} J_{\sigma} \supset K,
$$

then $\bigcup_{\sigma: J_{\sigma} \in \widetilde{\mathcal{J}}_{r}} G_{\sigma} \supset K_{r}$. Since $\overline{\text { int } J}=J \neq \emptyset, J$ contains a ball with a positive radius. Since $\delta_{1} r \leq\left|J_{\sigma}\right|<r$ and $J_{\sigma}$ is geometrically similar to $J$, there exists an integer $N_{1} \geq 1$ such that $N_{1} \mathscr{L}^{d}\left(J_{\sigma}\right) \geq \mathscr{L}^{d}\left(G_{\sigma}\right)$ for any $r>0$ and for any $J_{\sigma} \in \widetilde{J}_{r}$. Since $\mathscr{L}^{d}\left(J_{\sigma}\right) \leq \frac{r^{d} \mathscr{L}^{d}(J)}{|J|^{d}}$, we have

$$
\mathscr{L}^{d}\left(K_{r}\right) \leq \mathscr{L}^{d}\left(\bigcup_{\sigma: J_{\sigma} \in \tilde{\mathcal{J}}_{r}} G_{\sigma}\right) \leq M_{r}(K) r^{d} C_{d}^{\prime},
$$

where $C_{d}^{\prime}=\frac{N_{1} \mathscr{L}^{d}(J)}{|J|^{d}}$. Thus

$$
\limsup _{r \rightarrow 0} \frac{\log M_{r}(K)}{-\log r} \geq d-\liminf _{r \rightarrow 0} \frac{\log \mathscr{L}^{d}\left(K_{r}\right)}{\log r} .
$$

As a consequence of (7) and (8), the equality (6) must hold.

From Propositions 3.2 and 3.3, we have the following proposition.

\section{Proposition 3.4.}

$$
\overline{\operatorname{dim}}_{\mathcal{J}}(K)=\overline{\operatorname{dim}}_{B}(K) .
$$

From now on, to obtain the upper box dimension of the generalized random Moran set, it is equivalent to compute the upper $J$-packing dimension of $K(\omega)$.

\section{Proof of Theorem A}

Without loss of generality, in what follows, we always assume $|J|=1$. To prove Theorem A, we first list the following two facts (for its proof see Lemma 4.3 and Lemma 4.4 in [15]).

Lemma 4.1. Let $\mathcal{J}$ be a random Moran construction. Then there exists a positive constant $M>0$ depending on $\delta_{1}$ such that $n_{k} \leq M$ for any $k \geq 1$. 
Lemma 4.2. Let $\mathcal{J}$ be a random Moran construction. Then there exists a positive real number $\delta_{2}<1$ such that for any $k \geq 0$ and $\sigma \in I_{k}$, we have

$$
\max _{1 \leq i \leq n_{k+1}} T_{\sigma * i} \leq \delta_{2} \quad \text { a.e. }
$$

Next, we shall prove that

$$
\overline{\operatorname{dim}}_{\mathcal{J}}(K(\omega))=s^{*} \text { a.e., }
$$

where $\mathbb{E} \sum_{\sigma \in I_{k}}\left|J_{\sigma}\right|^{s_{k}}=1$ and $s^{*}=\lim _{\sup _{k \rightarrow \infty}} s_{k}$.

Proposition 4.3 (Upper bound estimation of $\overline{\operatorname{dim}}_{\mathcal{J}}(K(\omega))$ ). Let $\mathcal{J}$ be a generalized random Moran construction and $K(\omega)$ a generalized random Moran set associated with $\mathcal{J}$. Then for almost all $\omega \in \Omega$,

$$
\overline{\operatorname{dim}}_{\mathcal{J}}(K(\omega)) \leq s^{*}
$$

Proof. It suffices to prove that for any $t>s^{*}, \overline{\operatorname{dim}}_{\mathcal{J}}(K(\omega)) \leq t$ for almost all $\omega \in \Omega$. By contradiction, suppose that there exists a subset $A$ of $\Omega$ with $P(A)>0$ such that $\overline{\operatorname{dim}}_{J}(K(\omega))>t$ for any $\omega \in A$. Take a real number $s^{*}<\gamma<t$. By the definition of $s^{*}$, there exists $k_{0} \in \mathbb{N}$ such that for any $k>k_{0}$, we have $\gamma>s_{k}$. Hence $\mathbb{E}\left[\sum_{\tau \in I_{k}}\left|J_{\tau}(\omega)\right|^{\gamma}\right]<1$ for any $k>k_{0}$. On the other hand, by the definition of the upper $J$-packing dimension,

$$
\mathcal{P}_{\mathcal{J}}^{t}(K(\omega))=\limsup _{r \rightarrow 0} \sum_{\sigma: J_{\sigma}(\omega) \in \widetilde{\mathcal{J}}_{r}(\omega)}\left|J_{\sigma}(\omega)\right|^{t}>1
$$

for all $\omega \in A$. By the definition of $\delta_{1},\left|J_{\sigma}(\omega)\right| \geq \delta_{1}^{m}$ for any positive integer $m$ and any $\sigma \in I_{m}$. Then for any $r<\delta_{1}^{m}$ and any $J_{\sigma}(\omega) \in \widetilde{J}_{r}(\omega)$, we have

$$
J_{\sigma}(\omega) \in \bigcup_{k \geq m} \bigcup_{\tau \in I_{k}} J_{\tau}(\omega)
$$

since $\left|J_{\sigma}(\omega)\right| \leq r$. Therefore for any $r<\delta_{1}^{m}$,

$$
\sum_{\sigma: J_{\sigma}(\omega) \in \widetilde{\mathcal{J}}_{r}(\omega)}\left|J_{\sigma}(\omega)\right|^{t} \leq \sum_{k \geq m} \sum_{\tau \in I_{k}}\left|J_{\tau}(\omega)\right|^{t}
$$

and hence

$$
1<\limsup _{r \rightarrow 0} \sum_{\sigma: J_{\sigma}(\omega) \in \widetilde{\mathcal{J}}_{r}(\omega)}\left|J_{\sigma}(\omega)\right|^{t} \leq \limsup _{m \rightarrow \infty} \sum_{k \geq m} \sum_{\tau \in I_{k}}\left|J_{\tau}(\omega)\right|^{t}
$$

for any $\omega \in A$. Write $Q_{m}(\omega)=\sum_{k \geq m} \sum_{\tau \in I_{k}}\left|J_{\tau}(\omega)\right|^{t}$. By Lemma 4.2, we have

$$
\mathbb{E}\left[Q_{m}\right]=\sum_{k \geq m} \mathbb{E}\left[\sum_{\tau \in I_{k}}\left|J_{\tau}\right|^{\gamma}\left|J_{\tau}\right|^{t-\gamma}\right] \leq \sum_{k \geq m} \delta_{2}^{k(t-\gamma)} \mathbb{E}\left[\sum_{\tau \in I_{k}}\left|J_{\tau}\right|^{r}\right] \leq \sum_{k \geq m} \delta_{2}^{k(t-\gamma)}
$$

for any $m \geq k_{0}$. Since $0<\delta_{2}<1$ and $Q_{m}(\omega)$ is non-increasing, we can obtain that

which is a contradiction to (10).

$$
\lim _{m \rightarrow \infty} \mathbb{E}\left[Q_{m}(\omega)\right]=0
$$


Thus for any $t>s^{*}$ and almost all $\omega \in \Omega$, we have $\mathcal{P}_{\mathcal{J}}^{t}(K(\omega))<\infty$. Therefore

$$
\overline{\operatorname{dim}}_{\mathcal{J}}(K(\omega)) \leq s^{*} \quad \text { a.s. }
$$

From now on, we will estimate the lower bound of $\overline{\operatorname{dim}}_{B}(K(\omega))$. The key point is to prove that for any $\alpha<s^{*}$,

$$
P\left\{\omega: \limsup _{m \rightarrow \infty} \sum_{\sigma \in I_{m}}\left|J_{\sigma}(\omega)\right|^{\alpha}>0\right\}=1 .
$$

First, we give the following two simple facts which will be used later.

Lemma 4.4. For any $x \in[0,1), \log (1-x) \leq-x$.

Lemma 4.5. Let $0<\alpha<s^{*}$ and $\varepsilon=\frac{s^{*}-\alpha}{2}$. Let $0<\delta_{2}<1$ be defined as in Lemma 4.2. Then we have $e^{-x} \leq 1-\delta_{2}^{\varepsilon} x$ when $x$ is small enough.

The following proposition is an estimate of negative moments.

Proposition 4.6. Let $0<\alpha<s^{*}$ and $\varepsilon=\frac{s^{*}-\alpha}{2}$. Let $0<\delta_{2}<1$ be defined as in Lemma 4.2 and $u>0$ be small enough. Let

$$
R_{m+1}(u)=\mathbb{E}\left(e^{-u \sum_{\sigma \in I_{m+1}}\left|J_{\sigma}\right|^{\alpha}}\right) .
$$

Then

where $A_{m+1}=\mathbb{E}\left(\sum_{i=1}^{n_{m+1}} T_{\sigma * i}^{\alpha}\right)$ for any $\sigma \in I_{m}$.

$$
R_{m+1}(u) \leq R_{m}\left(u \delta_{2}^{\varepsilon} A_{m+1}\right),
$$

Proof. Since the random vectors $\left\{\left(T_{\sigma * 1}, \ldots, T_{\sigma * n_{m+1}}\right): \sigma \in I_{m}\right\}$ have the same distribution, $\mathbb{E}\left(\sum_{i=1}^{n_{m+1}} T_{\sigma * i}^{\alpha}\right)$ is a constant for any $\sigma \in I_{m}$. Since random vectors $\left\{\left(T_{\sigma * 1}, \ldots, T_{\sigma * n_{|\sigma|+1}}\right): \sigma \in I_{*}\right\}$ are independent,

$$
\begin{aligned}
R_{m+1}(u) & =\mathbb{E}\left(e^{-u \sum_{\sigma \in I_{m+1}}\left|J_{\sigma}\right|^{\alpha}}\right)=\mathbb{E}\left(e^{-u \sum_{\sigma \in I_{m}}\left(\left|J_{\sigma}\right|^{\alpha} \sum_{i=1}^{n_{m}+1} T_{\sigma * i}^{\alpha}\right)}\right) \\
& =\mathbb{E}\left(\prod_{\sigma \in I_{m}} e^{-u\left|J_{\sigma}\right|^{\alpha} \sum_{i=1}^{n_{m}+1} T_{\sigma * i}^{\alpha}}\right)=\prod_{\sigma \in I_{m}} \mathbb{E}\left(e^{-u\left|J_{\sigma}\right|^{\alpha} \sum_{i=1}^{n_{m}+1} T_{\sigma * i}^{\alpha}}\right) .
\end{aligned}
$$

By Lemma 4.1 and Lemma 4.2, there exists a constant $C$ such that $\sum_{i=1}^{n_{|\sigma|+1}} T_{\sigma * i}^{\alpha}<$ $C$ for any $\sigma \in I_{*}$ almost surely. Since $\left|J_{\sigma}\right| \leq 1$ for any $\sigma \in I_{*}$, then we have, when $u>0$ is small enough,

$$
0<u\left|J_{\sigma}\right|^{\alpha} \sum_{i=1}^{n_{|\sigma|+1}} T_{\sigma * i}^{\alpha} \leq C u
$$

for any $\sigma \in I_{*}$ almost surely. And then by Lemma 4.5 and the independence of random vectors, for any $\sigma \in I_{m}$,

$$
\mathbb{E}\left(e^{-u\left|J_{\sigma}\right|^{\alpha} \sum_{i=1}^{n_{m}+1} T_{\sigma * i}^{\alpha}}\right) \leq \mathbb{E}\left(1-\delta_{2}^{\varepsilon} u\left|J_{\sigma}\right|^{\alpha} \sum_{i=1}^{n_{m+1}} T_{\sigma * i}^{\alpha}\right)
$$




$$
\begin{aligned}
& =1-\delta_{2}^{\varepsilon} u \mathbb{E}\left(\left|J_{\sigma}\right|^{\alpha} \sum_{i=1}^{n_{m+1}} T_{\sigma * i}^{\alpha}\right) \\
& =1-\delta_{2}^{\varepsilon} u \mathbb{E}\left(\left|J_{\sigma}\right|^{\alpha}\right) A_{m+1} \\
& =\mathbb{E}\left(1-\delta_{2}^{\varepsilon} u\left|J_{\sigma}\right|^{\alpha} A_{m+1}\right),
\end{aligned}
$$

where $A_{m+1}=\mathbb{E}\left(\sum_{i=1}^{n_{m+1}} T_{\sigma * i}^{\alpha}\right)$. Hence, by the independence of random vectors and Lemma 4.4,

$$
\begin{aligned}
R_{m+1}(u) & \leq \prod_{\sigma \in I_{m}} \mathbb{E}\left(1-\delta_{2}^{\varepsilon} u\left|J_{\sigma}\right|^{\alpha} A_{m+1}\right) \\
& =\mathbb{E}\left[\prod_{\sigma \in I_{m}}\left(1-\delta_{2}^{\varepsilon} u\left|J_{\sigma}\right|^{\alpha} A_{m+1}\right)\right] \\
& =\mathbb{E}\left[e^{\log \prod_{\sigma \in I_{m}}\left(1-\delta_{2}^{\varepsilon} u\left|J_{\sigma}\right|^{\alpha} A_{m+1}\right)}\right] \\
& =\mathbb{E}\left[e^{\sum_{\sigma \in I_{m}} \log \left(1-\delta_{2}^{\varepsilon} u\left|J_{\sigma}\right|^{\alpha} A_{m+1}\right)}\right] \\
& \leq \mathbb{E}\left(e^{-\sum_{\sigma \in I_{m}} \delta_{2}^{\varepsilon} u\left|J_{\sigma}\right|^{\alpha} A_{m+1}}\right) \\
& =R_{m}\left(u \delta_{2}^{\varepsilon} A_{m+1}\right),
\end{aligned}
$$

which completes the proof.

Remark that from the proof of Proposition 4.6, we know that if $u$ is small than an absolute constant, then the proposition follows for any $m \geq 1$.

Proposition 4.7. Let $0<\alpha<s^{*}$. And let

$$
W(\omega)=\limsup _{m \rightarrow \infty} \sum_{\sigma \in I_{m}}\left|J_{\sigma}(\omega)\right|^{\alpha} .
$$

Then

$$
P\{W>0\}=1 .
$$

Proof. For any $0<\alpha<s^{*}$, let $\varepsilon=\frac{s^{*}-\alpha}{2}$. By the definition of $s^{*}$, there exists a subsequence $\left\{m_{k}\right\} \uparrow \infty$ such that $s_{m_{k}} \rightarrow s^{*}$ and $0<\alpha<s_{m_{k}}<\alpha+\varepsilon$ for all $k=0,1,2, \ldots$. Moreover,

$$
\mathbb{E}\left(\sum_{\sigma \in I_{m_{k}}}\left|J_{\sigma}\right|^{\alpha+\varepsilon}\right)>\mathbb{E}\left(\sum_{\sigma \in I_{m_{k}}}\left|J_{\sigma}\right|^{s_{m_{k}}}\right)=1 .
$$

Using Proposition 4.6, for any $u>0$ small enough,

$$
\begin{aligned}
R_{m_{k}}(u) & \leq R_{m_{k}-1}\left(u \delta_{2}^{\varepsilon} A_{m_{k}}\right) \leq R_{m_{k}-2}\left(u \delta_{2}^{2 \varepsilon} A_{m_{k}} A_{m_{k}-1}\right) \leq \cdots \\
& \leq R_{m_{0}}\left(u \delta_{2}^{\left(m_{k}-m_{0}\right) \varepsilon} A_{m_{k}} \cdots A_{m_{0}+1}\right) \\
& =\mathbb{E}\left[\exp \left\{-u \delta_{2}^{\left(m_{k}-m_{0}\right) \varepsilon} A_{m_{k}} \cdots A_{m_{0}+1} \sum_{\sigma \in I_{m_{0}}}\left|J_{\sigma}\right|^{\alpha}\right\}\right] .
\end{aligned}
$$


Since random vectors $\left\{\left(T_{\sigma * 1}, \ldots, T_{\sigma * n_{|\sigma|+1}}\right): \sigma \in I_{*}\right\}$ are independent and $\left\{\left(T_{\sigma * 1}, \ldots, T_{\sigma * n_{k+1}}\right): \sigma \in I_{k}\right\}$ have the same distribution for every $k \geq 0$,

$$
A_{m_{k}} \cdots A_{m_{0}+1}=\frac{\mathbb{E} \sum_{\sigma \in I_{m_{k}}}\left|J_{\sigma}\right|^{\alpha}}{\mathbb{E} \sum_{\sigma \in I_{m_{0}}}\left|J_{\sigma}\right|^{\alpha}}
$$

Thus

$$
\begin{aligned}
R_{m_{k}}(u) & \leq \mathbb{E}\left[\exp \left\{-u \delta_{2}^{\left(m_{k}-m_{0}\right) \varepsilon} A_{m_{k}} \cdots A_{m_{0}+1} \sum_{\sigma \in I_{m_{0}}}\left|J_{\sigma}\right|^{\alpha}\right\}\right] \\
& =\mathbb{E}\left[\exp \left\{-u \delta_{2}^{\left(m_{k}-m_{0}\right) \varepsilon} \frac{\mathbb{E} \sum_{\sigma \in I_{m_{k}}}\left|J_{\sigma}\right|^{\alpha}}{\mathbb{E} \sum_{\sigma \in I_{m_{0}}}\left|J_{\sigma}\right|^{\alpha}} \sum_{\sigma \in I_{m_{0}}}\left|J_{\sigma}\right|^{\alpha}\right\}\right] .
\end{aligned}
$$

By Lemma 4.2 and the inequality (11),

$$
\mathbb{E} \sum_{\sigma \in I_{m_{k}}}\left|J_{\sigma}\right|^{\alpha}=\mathbb{E}\left(\sum_{\sigma \in I_{m_{k}}}\left|J_{\sigma}\right|^{\alpha+\epsilon}\left|J_{\sigma}\right|^{-\epsilon}\right) \geq \delta_{2}^{-m_{k} \epsilon} \mathbb{E} \sum_{\sigma \in I_{m_{k}}}\left|J_{\sigma}\right|^{\alpha+\epsilon} \geq \delta_{2}^{-m_{k} \epsilon} .
$$

We obtain

$$
\begin{aligned}
R_{m_{k}}(u) & <\mathbb{E}\left[\exp \left\{-u \delta_{2}^{\left(m_{k}-m_{0}\right) \varepsilon} \delta_{2}^{-m_{k} \varepsilon} \frac{\sum_{\sigma \in I_{m_{0}}}\left|J_{\sigma}\right|^{\alpha}}{\mathbb{E} \sum_{\sigma \in I_{m_{0}}}\left|J_{\sigma}\right|^{\alpha}}\right\}\right] \\
& =\mathbb{E}\left[\exp \left\{-u \delta_{2}^{-m_{0} \epsilon} \frac{\sum_{\sigma \in I_{m_{0}}}\left|J_{\sigma}\right|^{\alpha}}{\mathbb{E} \sum_{\sigma \in I_{m_{0}}}\left|J_{\sigma}\right|^{\alpha}}\right\}\right] .
\end{aligned}
$$

By Lemma 4.1 and Lemma 4.2, we have

$$
2^{m_{0}} \delta_{1}^{m_{0} \alpha} \leq \sum_{\sigma \in I_{m_{0}}}\left|J_{\sigma}\right|^{\alpha} \leq M^{m_{0}} \delta_{2}^{m_{0} \alpha}
$$

Thus

$$
\begin{aligned}
R_{m_{k}}(u) & \leq \mathbb{E}\left[\exp \left\{-u \delta_{2}^{-m_{0} \epsilon} \frac{\sum_{\sigma \in I_{m_{0}}}\left|J_{\sigma}\right|^{\alpha}}{\mathbb{E} \sum_{\sigma \in I_{m_{0}}}\left|J_{\sigma}\right|^{\alpha}}\right\}\right] \\
& \leq \mathbb{E}\left[\exp \left\{-u \delta_{2}^{-m_{0} \varepsilon} \frac{2^{m_{0}} \delta_{1}^{m_{0} \alpha}}{M^{m_{0}} \delta_{2}^{m_{0} \alpha}}\right\}\right]=\exp \left\{-u \delta_{2}^{-m_{0} \varepsilon} \frac{2^{m_{0}} \delta_{1}^{m_{0} \alpha}}{M^{m_{0}} \delta_{2}^{m_{0} \alpha}}\right\}<1 .
\end{aligned}
$$

Therefore, for any $u>0$,

$$
\begin{aligned}
P\{W=0\} & \leq \mathbb{E}\left(e^{-u W}\right)=\mathbb{E}\left(e^{-u \limsup _{m \rightarrow \infty} \sum_{\sigma \in I_{m}}\left|J_{\sigma}\right|^{\alpha}}\right) \\
& =\mathbb{E}\left(\liminf _{m \rightarrow \infty} e^{-u \sum_{\sigma \in I_{m}}\left|J_{\sigma}\right|^{\alpha}}\right) \\
& \leq \liminf _{m \rightarrow \infty} \mathbb{E}\left(e^{-u \sum_{\sigma \in I_{m}}\left|J_{\sigma}\right|^{\alpha}}\right) \\
& =\liminf _{m \rightarrow \infty} R_{m}(u) \leq \liminf _{k \rightarrow \infty} R_{m_{k}}(u)<1 .
\end{aligned}
$$

This implies that $P\{W>0\}>0$. By Kolmogorov 0-1 law, we obtain that $P\{W>0\}=1$. 
Proposition 4.8 (Lower bound estimation of $\overline{\operatorname{dim}}_{\mathcal{J}}(K(\omega))$ ). Let $\mathcal{J}$ be a generalized Moran construction and $K(\omega)$ be a generalized Moran set associated with $\mathcal{J}$. Then for almost all $\omega \in \Omega$,

$$
\overline{\operatorname{dim}}_{\mathcal{J}}(K(\omega)) \geq s^{*} .
$$

Proof. It suffices to prove that for any $0<\beta<s^{*}, \overline{\operatorname{dim}}_{\mathcal{J}}(K(\omega)) \geq \beta$ for almost all $\omega \in \Omega$. By Proposition 4.7, for any $0<\beta<\alpha<s^{*}$,

$$
\limsup _{m \rightarrow \infty} \sum_{\sigma \in I_{m}}\left|J_{\sigma}(\omega)\right|^{\alpha}>0
$$

for almost all $\omega \in \Omega$. We shall prove that for almost all $\omega \in \Omega$,

$$
\overline{\operatorname{dim}}_{\mathcal{J}}(K(\omega)) \geq \beta \text {. }
$$

In fact, for those $\omega \in \Omega$ satisfying the inequality (12), define

$$
c(\omega)=\min \left\{\limsup _{m \rightarrow \infty} \sum_{\sigma \in I_{m}}\left|J_{\sigma}(\omega)\right|^{\alpha}, 1\right\} .
$$

Then there exist infinitely many integers $m$ such that

$$
\sum_{\sigma \in I_{m}}\left|J_{\sigma}(\omega)\right|^{\alpha}>\frac{c(\omega)}{2} .
$$

The following proofs are similar to that in the second part of Proposition 3.2. Let $n_{k}$ be the number of $\sigma \in I_{m}$ such that $\delta_{2}^{k+1}<\left|J_{\sigma}(\omega)\right| \leq \delta_{2}^{k}$. We claim that there exists some integer $k_{m}$ such that $n_{k_{m}} \geq \delta_{2}^{-k_{m} \beta}\left(1-\delta_{2}^{\alpha-\beta}\right) \frac{c(\omega)}{2}$. Otherwise, $n_{k}<\delta_{2}^{-k \beta}\left(1-\delta_{2}^{\alpha-\beta}\right) \frac{c(\omega)}{2}$ for any $k$. Then

$$
\sum_{\sigma \in I_{m}}\left|J_{\sigma}(\omega)\right|^{\alpha} \leq \sum_{k \geq 0} \delta_{2}^{-k \beta}\left(1-\delta_{2}^{\alpha-\beta}\right) \frac{c(\omega)}{2} \delta_{2}^{k \alpha}=\frac{c(\omega)}{2},
$$

a contradiction to (13). Let $M_{\delta_{2}^{k m}}(K(\omega))$ denote the number of the sets $J_{\sigma} \in$ $\widetilde{J}_{\delta_{2}^{k m}}$. Then

$$
M_{\delta_{2}^{k m}}(K(\omega)) \geq n_{k_{m}} \geq \delta_{2}^{-k_{m} \beta}\left(1-\delta_{2}^{\alpha-\beta}\right) \frac{c(\omega)}{2},
$$

which implies $\overline{\operatorname{dim}}_{\mathcal{J}}(K(\omega)) \geq \beta$. Thus the conclusion follows.

From Proposition 3.4, Proposition 4.3 and Proposition 4.8, we have

$$
\overline{\operatorname{dim}}_{B}(K(\omega))=\overline{\operatorname{dim}}_{\mathcal{J}}(K(\omega))=s^{*} \text { a.e. }
$$

By the equality (14), to prove Theorem A, it suffices to prove the following proposition.

Proposition 4.9. Let $\mathcal{J}$ be a generalized Moran construction and $K(\omega)$ be a generalized Moran set associated with $\mathcal{J}$. Then for almost all $\omega \in \Omega$,

$$
\operatorname{dim}_{P}(K(\omega))=\overline{\operatorname{dim}}_{B}(K(\omega)) .
$$


Proof. First, we shall prove that for any $\sigma \in I_{*}$,

$$
\overline{\operatorname{dim}}_{B}\left(J_{\sigma}(\omega) \cap K(\omega)\right)=\overline{\operatorname{dim}}_{\mathcal{J}}\left(J_{\sigma}(\omega) \cap K(\omega)\right)=s^{*} \text { a.e. }
$$

Since $J_{\sigma}(\omega) \cap K(\omega)$ is also a generalized random Moran set, by the equality (14),

$$
\overline{\operatorname{dim}}_{B}\left(J_{\sigma}(\omega) \cap K(\omega)\right)=\overline{\operatorname{dim}}_{\mathcal{J}}\left(J_{\sigma}(\omega) \cap K(\omega)\right)=s^{\prime} \text { a.e. },
$$

where $s_{k}^{\prime}$ satisfies the equation

$$
\mathbb{E} \sum_{\tau \in I_{k}} \prod_{i=|\sigma|+1}^{|\sigma|+k} T_{\sigma \tau \mid i}^{s_{k}^{\prime}}=1,
$$

and $s^{\prime}=\lim \sup _{k \rightarrow \infty} s_{k}^{\prime}$. Remark that $\delta_{1} \leq T_{\tau} \leq \delta_{2}$ for any $\emptyset \neq \tau \in I_{*}$ and that for each $k \geq 0$, the vectors $\left\{\left(T_{\sigma * 1}, \ldots, T_{\sigma * n_{k+1}}\right): \sigma \in I_{k}\right\}$ have the same distribution. Thus comparing the equations (1) and (15) yields $s^{\prime}=s^{*}$. So there exists a set $A \subset \Omega$ with $P(A)=1$ such that for any $\omega \in A$ and any $\sigma \in I_{*}$,

$$
\overline{\operatorname{dim}}_{B}\left(J_{\sigma}(\omega) \cap K(\omega)\right)=\overline{\operatorname{dim}}_{B}(K(\omega)) .
$$

For any $\omega \in A$ and any open set $V$ satisfying $V \cap K(\omega) \neq \emptyset$, there exists some $\sigma \in I_{*}$ such that $J_{\sigma}(\omega) \subset V \cap K(\omega)$. Then

$$
\overline{\operatorname{dim}}_{B}(K(\omega))=\overline{\operatorname{dim}}_{B}\left(J_{\sigma}(\omega) \cap K(\omega)\right) \leq \overline{\operatorname{dim}}_{B}(V \cap K(\omega)) \leq \overline{\operatorname{dim}}_{B}(K(\omega)),
$$

thus $\overline{\operatorname{dim}}_{B}(K(\omega))=\overline{\operatorname{dim}}_{B}(V \cap K(\omega))$. So by Lemma 2.1, the conclusion follows.

Theorem A follows from Proposition 4.9 and the equality (14).

Acknowledgements. The authors are grateful to Professor In-Soo Baek with whom we have discussed the problem of this paper. The research was supported by NSFC 11171124 and NSFC 11026171.

\section{References}

[1] M. Arbeiter, Random recursive construction of self-similar fractal measure. The noncompact case, Probab. Theory Related Fields 88 (1991), no. 4, 497-520.

[2] M. Arbeiter and N. Patzschke, Random self-similar multifractals, Math. Nachr. 181 (1996), 5-42.

[3] K. J. Falconer, Random fractals, Math. Proc. Cambridge Philos. Soc. 100 (1986), no. 3, 559-582.

[4] , Fractal Geometry. Mathematical Foundation and Applications, John Wily and Sons, Ltd., Chichester, 1990.

[5] - Techniques in Fractal Geometry, John Wiley and Sons, Ltd. Chichester, 1997.

[6] S. Graf, Statistically self-similar fractals, Probab. Theory Related Fields 74 (1987), no. 3, 357-392.

[7] S. Graf, R. D. Mauldin, and S. C. Williams, The exact Hausdorff dimension in random recursive constructions, Mem. Amer. Math. Soc. 71 (1988), no. 381, x+121 pp.

[8] S. Hua, The dimensions of generalized self-similar sets, Acta Math. Appl. Sin. 17 (1994), no. 4, 551-558.

[9] S. Hua and W. X. Li, Packing dimension of generalized Moran sets, Prog. Nat. Sci. 6 (1996), no. 2, 148-152. 
[10] S. Hua, H. Rao, Z. Y. Wen, and J. Wu, On the structures and dimensions of Moran sets, Sci. China Ser. A 43 (2000), no. 8, 836-852.

[11] J. E. Hutchinson, Fractals and self-similarity, Indiana Univ. Math. J. 30 (1981), no. 5, $713-747$.

[12] J. E. Hutschinson and L. Ruschendorff, Random fractal measures via the contraction method, Indiana Univ. Math. J. 47 (1998), no. 2, 471-487.

[13] Y. Kifer, Fractals via Random Iterated Function Systems and Random Geometric Constructions, Fractal Geometry and Stochastics (Finsterbergen, 1994), pp. 145-164; Progr. Probab. 37 Birkhäuser, Basel, 1995.

[14] _ Fractal dimensions and random transformations, Trans. Amer. Math. Soc. 348 (1996), no. 5, 2003-2038.

[15] Y. Y. Liu, Z. Y. Wen, and J. Wu, Generalized random recursive constructions and geometric properties of random fractals, Math. Nachr. 267 (2004), 65-76.

[16] R. D. Mauldin and S. C. Williams, Random recursive constructions: asymptotic geometric and topological properties, Trans. Amer. Math. Soc. 295 (1986), no. 1, 325-346.

[17] P. A. P. Moran, Additive functions of intervals and Hausdorff measure, Proc. Cambridge Philos. Soc. 12 (1946), 15-23.

[18] L. Olsen, Random geometrically graph directed self-similar multifractals, Pitman Research Notes in Mathematics Series, 307. Longman Scientific and Technical, Harlow; copublished in the United States with John Wiley \& Sons, Inc., New York, 1994

[19] N. Patzschke and M. Zähle, Self-similar random measures are locally scale invariant, Probab. Theory Related Fields 97 (1993), no. 4, 559-574.

[20] N. Patzschke and U. Zähle, Self-similar random measures IV. The recursive construction model of Falconer, Graf, and Mauldin and Williams, Math. Nachr. 149 (1990), 285-302.

[21] Y. Pesin, Dimension Theory in Dynamical Systems, University of Chicago Press, Chicago, IL, 1997.

[22] Y. Pesin and H. Weiss, On the dimension of deterministic and random Cantor-like sets, symbolic dynamics, and the Eckmann-Ruelle conjecture, Comm. Math. Phys. 182 (1996), no. 1, 105-153.

XIN TONG

Department of Mathematics

WUHAN UNIVERSITY

430072 Wuhan, P. R. China

E-mail address: tongxinxin@126.com

YUE-LI YU

Department of Mathematics

WUHAN UNIVERSITY

430072 Wuhan, P. R. China

E-mail address: yuyueli@whu.edu.cn

XiAO-JUN ZHAO

SCHOOL OF ECONOMICS

Peking University

100871 Peking, P. R. China

E-mail address: zhaoxiaojun@pku.edu.cn 